\title{
Creating space to build emotional resilience in the animal research community
}

\author{
The relationships between individuals and the research animals they work with can enhance animal welfare, \\ but they also involve a moral cost to staff. Securing a safe space to communicate openly about animal welfare \& \\ research and acknowledge its emotional impacts is crucial. In this Comment, we reflect on emotional resilience and \\ provide resources available to help manage the emotional burden of working with laboratory animals.
}

\section{Jordi L. Tremoleda and Angela Kerton}

T he recent Covid-19 pandemic has forced many institutions to scale back operations, including animal research. Institutions as well as suppliers, who have reported receiving fewer orders, have had to greatly reduce their animal stocks ${ }^{1}$. After being tasked with culling those stocks, animal staff must now support all the general maintenance of those animals that remain. While there is a general consensus among institutional governance that these decisions had to be taken to support 'stay at home' recommendations and reduce risk to human health, it is important that we acknowledge the emotional pressures of performing such actions.

For animal care staff, euthanasia remains a major professional duty with an enormous emotional burden. It is a regulatory requirement that laboratory animals be humanely euthanized to alleviate any unnecessary pain and suffering to protected animals, or when necessary as the result of a research study. While animal researchers may find some comfort from the fact that the animals they are euthanizing have a justifiable purpose and meaning and that the procedure is appropriately performed, facing the task of euthanizing animals can be psychologically challenging and lead to euthanasia stress. The tension between a commitment to keep healthy animals and the obligation to euthanize those same animals in a professional manner is one of the hardest elements of the job to reconcile ${ }^{2,3}$.

The relationship between laboratory animals and those who work with them has an overwhelming impact not just on the animals' wellbeing, but also on the emotional health of animal staff. This emotional impact is exacerbated by the responsibilities of working with other sentient beings and determining how best to ensure their wellbeing following humanely induced interventions that

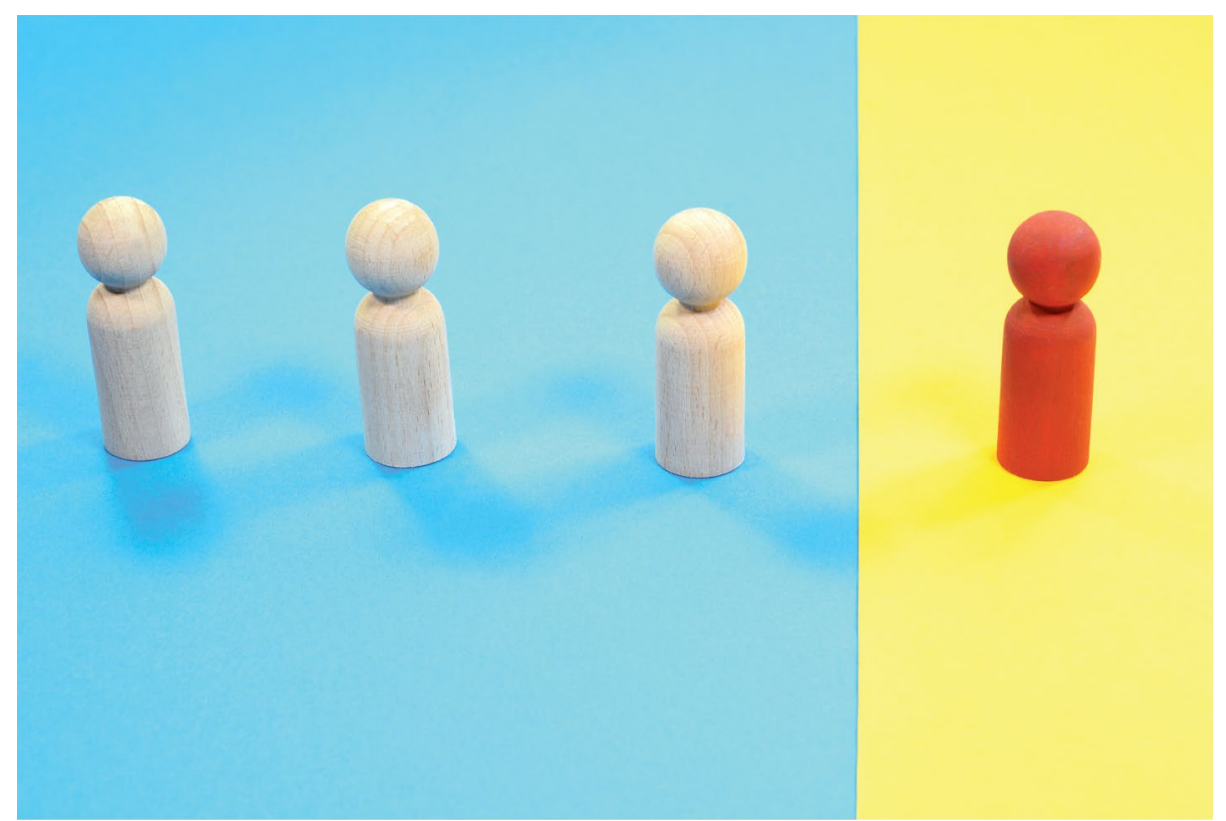

Compassion fatigue among animal staff can lead to feelings of isolation and hinder lab animal care. A supportive environment is key for building emotional resilience. Credit: jayk7 / Moment / Getty

cause certain degrees of harm or distress. Staff can also feel pressured by increasing social accountability ${ }^{4}$ and by their own individual moral values on care and compassion. The professional expectation that those working in animal research be devoid of concerns is not realistic, as all people are prone to stress because of the peculiarities of their duties 5 .

Indeed, staff working with laboratory animals are exposed to many moral stressors, including experimental procedures and animal euthanasia, that significantly contribute to the development of compassion fatigue, ${ }^{6}$ a condition characterized by a reduced capacity for empathy and caring behavior following the knowledge that others have experienced a traumatizing event. Compassion fatigue can drive physical and emotional distress and diminish the quality of care and welfare attention delivered to animals? ${ }^{7}$. Symptoms can include disruptive attitudes, such as lack of communication with colleagues and isolation from others, which can progress towards bottling up emotions, difficulty concentrating, mental and physical tiredness, and depression.

A related concept, empathetic distress fatigue, may better capture the consequences for caregivers such as animal staffs.

Compassion is an attitude associated with 'feeling for', while empathy is more closely associated with a sense of 'feeling with' and has a stronger affective impact'. Such an excessive emotional altruism, if not well balanced, can lead to an excess of selfresponsibilities; this pressure can progress 


\section{Table 1 | Emotional resilience on-line resources}

\begin{tabular}{l} 
Source \\
\hline The Royal College of Veterinary Surgeons (RCVS) \\
The Webinar Vet \\
The University of Oxford \\
UK mental health charity Mind \\
American Association for Laboratory Animal Science \\
(AALAS) \\
Canadian Association for Laboratory Animal Science \\
Association Canadienne pour la Science des Animaux \\
de Laboratoire (CALAS/ACSAL
\end{tabular}

LabRoots

Institute of Animal Technology, UK

\section{Resource}

Mind Matters Initiative (MMI) https://www.vetmindmatters.org/

'Turning mind-full to mindful' https://www.thewebinarvet.com/course/mindfulness-course-series-1/

Mindfulness Centre https://www.oxfordmindfulness.org/

https://www.mind.org.uk/

Cost of Caring https://www.aalas.org/education/educational-resources/cost-of-caring/

COVID-19: One person's perspective on the toll on animals and humans, from present state of the pandemic https://calas-acsal.org/site/resources/articles/march2

Compassion Fatigue: Education and Engagement in Animal Research by Marian Esvelt, DVM https://www.labroots.com/webinar/compassion-fatigue-education-engagement-animal-research/

Let's talk euthanasia https://tinyurl.com/letstakIAT1

It's OK, not to be OK, Let's talk about COVID-19 https://tinyurl.com/letstakIAT2 towards disconnected emotions and thus, less attention to animal care.

A good culture of care is key to the welfare of laboratory animals, and it is also central to the wellbeing of the staff working directly and indirectly with those animals; this in turn has a direct impact on the quality of the science ${ }^{10,11}$. Empathetic behavior can only develop when staff feel emotionally and physically safe and that they are valued; institutions must support this caring professional attitude and ensure that their staff perceive actions and communications from management as supportive.

Providing a safe space where the staff can reflect on their individual experiences and receive support for their emotional and/or physical challenges is important. Sharing and analyzing personal experiences at work, whether individually or in groups, can help others learn from those experiences and encourage an attitude of care. Team exploration and interactive reflection are also important, as individuals with different responsibilities will identify different issues that can affect behavior. Institutions should be acknowledging the affective state of their staff members and encourage their confidence in providing compassionate care. For example, well-recognized techniques for developing compassion skills are found in mindfulness programs, which provide personal space and tools to promote individual and group communication.

Engaging communication should occur between staff with different levels and areas of expertise, as one should be mindful of different moral or cultural values and competing pressures and responsibilities. It is important to appreciate the different dimensions and expressions of emotional resilience-and some of its challengesby reflecting on the ways in which the emotional labor of caring is distributed across all staff working in animal research.

\section{Resources to support cultures of care} and building up emotional resilience

Emotional resilience is the ability to respond and adapt to stressful situations. Whilst laboratory animal personnel may be particularly at risk of experiencing significant stress in their work, it is important that they can tackle such challenges and live through adversity in their caring duties. Being flexible and adaptable is crucial and everyone can take steps to develop greater personal emotional resilience.

Strategies for supporting care and emotional work include sharing responsibilities, sharing scientific work and goals, and sharing stories between the different stakeholders in animal research. Animal Welfare Bodies and Institutional Ethical Committees must reinforce the importance of shared responsibility and having a pro-active approach towards animal care and welfare; they should also underscore the importance of effective communication and valuing interpersonal elements of care to empower their staff and show that them they are respected, will be listened to, and that their roles are supported throughout the establishment. It is crucial that all voices and concerns be heard, emotional challenges recognized, and all dealt with positively.

Divide emotional labor. Promoting team working approaches with well-assigned individual and group-shared responsibilities remains an important strategy for nurturing a good culture of care; it can also help dilute individual emotional burdens. Such a collaborative ethos should be already established across all the animal research community, including reciprocal interaction between researchers and animal care staff. A hierarchic approach can be detrimental to openness and communication and could lead to staff disengagement, eventually evolving towards less caring attitudes ${ }^{2,5}$. The division of labor is well aligned to efficiency of labor, and it is likely to reduce the impact of challenging duties, such as culling and euthanasia, thus serving to minimize staff stress and anxiety.

Support emotional openness. Laboratory animal set ups, which can often feel very formal and focused on compliance and biosafety regulations, can make displaying emotions complex. Common areas are generally dedicated to catering or bench administrative duties, with limited social space and generally restricted natural lightning. However, it is important to provide both individuals and teams with a space to accommodate 'switching off' for personal and reflective time and where staff can feel comfortable openly discussing their work and its emotional impact.

Communication during breaks must be encouraged and that time protected. Staff can easily develop a protective attitude towards emotional 'old wounds;' such seemingly emotional resilience can easily build up to disconnection and lack of engagement. Furthermore, laboratory animal professionals often do not talk openly in public about their chosen career pathway for fear of disproval or personal security concerns. This may lead to feelings of suppression and even shame for working in a somewhat 'covert', little understood professional sector. Therefore, we need to acknowledge emotions and facilitate openness within a safe and respectful space in the lab animal field. 
While it is true the provision of many professional and environmental working factors are directly impacted by institutional and managerial decisions (e.g. working rotas, scheduling, work expectation and appraisal, delivery outcomes, working physical space, sick /annual leave...), managers are not professional mental health care workers, nor do they have the specific training for undertaking such responsibilities. Therefore it is important that institutions facilitate access to professional counselling and pastoral support, and that staff are well informed of these opportunities. Nevertheless, managers may find themselves the first port of call for those in need of support, so it is important that the appropriate routes of communication are established, to preserve trust and confidentiality with the managers and staff.

\section{Be aware of the increasing resources} available. Individual pro-action remains one of the key pillars of emotional balance, along with the abovementioned approaches to express emotional challenges and to develop better coping mechanisms. Techniques that encourage expression and openness of emotions and support positive learning from personal experiences to build up optimistic patterns and judgements on specific challenges would promote better emotional connection with colleagues. Similarly, supporting personal acceptance and avoiding comparison with others while building up pride in any job, small or large, will enhance participation and engagement among colleagues. There are many professional resources available to support this, particularly for animal technologists ${ }^{12,13}$, and they adapt to a range of new working measures while also highlighting the need to protect work/life balance. Several resources are summarized in Table 1.

The laboratory animal field is facing considerable challenges, in light of the Covid-19 pandemic and beyond it, with increasing expectations for new therapeutic discoveries and stronger commitments to protect the welfare of both animals and laboratory animal workers. As these challenging demands increase, so does the risk of compassion \& empathetic fatigue and the need to protect the emotional integrity of animal staff. Support remains limited, and we urge readers to continue raising awareness and developing resources to encourage a culture of openness regarding emotional labor and the support of staff dealing with compassion fatigue. The implementation of discussion platforms and resilience training opportunities that we have identified will improve not only animal welfare and staff wellbeing, but also the integrity of our research.

In other words: "Sharing science, sharing care."

Jordi L. Tremoleda (D) $1,2 \bowtie$ and Angela Kerton ${ }^{3}$
${ }^{1}$ The Blizard Institute, Barts and the London School
of Medicine, London, UK. ${ }^{2}$ Biological Services.
4 Newark St, London E1 2AT, UK. ${ }^{3}$ The Learning
Curve (Development) Ltd., P.O Box 140, Ware,
Hertfordshire SG90ZN, UK.
${ }_{\text {e-mail: j.lopez-tremoleda@qmul.ac.uk }}$

${ }^{1}$ The Blizard Institute, Barts and the London School of Medicine, London, UK. ${ }^{2}$ Biological Services. Curve (Development) Ltd, P.OB Box 140, Ware,

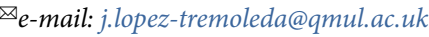

Published online: 31 August 2020

https://doi.org/10.1038/s41684-020-0637-7

References

1. Grimm, D. Science. https://doi.org/10.1126/science.abb8633 (2020).

2. Davies, K. \& Lewis, D. Anim Technol Welfare 9, 1-6 (2010).

3. AALAS. Compassion Fatigue: The Cost of Caring. Human emotions in the care of laboratory animals. https://www.aalas.org/ education/educational-resources/cost-of-caring [Accessed July 2020]

4. Ipsos MORI. Attitudes to animal research in 2016. https://www. ipsos.com/ipsos-mori/en-uk/attitudes-animal-research-2016 (2016). [Accessed July 2020]

5. Davies, K. Emotional Dissonance among UK Animal Technologists: Evidence, Impact and Management Implications. PhD Thesis. https://pearl.plymouth.ac.uk/handle/10026.1/3086 (2013). [Accessed July 2020]

6. Compassion Fatigue Awareness Project. Recognising compassion fatigue. http://www.compassionfatigue.org/pages/symptoms.html (2017). [Accessed July 2020]

7. Scotney, R. L., McLaughlin, D. \& Keates, H. L. J Am Vet Med Assoc 247, 1121-1130, https://doi.org/10.2460/javma.247.10.1121 (2015).

8. Newsome, J. T., Clemmons, E. A. \& Fitzhugh, D. C. et al. J Am Assoc Lab Anim Sci. 58, 289-292 (2019). https://doi.org/ 10.30802\%2FAALAS-JAALAS-18-000092

9. Klimecki, O., Singer, T. Empathic distress fatigue rather than compassion fatigue? Integrating findings from empathy research in psychology and social neuroscience. In: Oakley B, Knafo A, Madhavan G, et al. eds. Pathological Altruism. New York, New York: Oxford University Press, 1-23 (2011).

10. P Greenhough, B. \& Roe, E. Sci Technol Human Values. 43, 694-722 (2018). https://doi.org/10.1177\%2F0162243917718066.

11. P, Hawkins. Communication and the Culture of Care. Conference: Culture of Care Network. (2018). https://doi.org/10.13140/ RG.2.2.33865.80481

12. Institute of Animals Technology. It's OK ... NOT TO BE OKAY ...Let's Talk Coping with Change ... A Future of Hope ...? https:// www.iat.org.uk/ [Accessed July 2020]

13. Institute of Animal Technology (IAT) Let's talk euthanasia (2020). https://www.iat.org.uk/ [Accessed July 2020]

Acknowledgements

We are very grateful to Dr Beth Greenhough for her guidance and support during the writing of this document.

The authors declare no competing interests.

\title{
Taking an interest in small animal research programs
}

\author{
There are administrative challenges inherent to any animal research program - from hiring vets and care \\ staff to approving and overseeing protocols to reporting to the relevant authorities. Small institutions, with \\ limited resources and available staff compared to 'research juggernauts,' can have extra hurdles to cross when \\ administering their animal research programs.
}

\section{Christopher S. Keator}

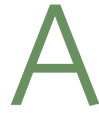
nyone who's attended an IACUC ${ }^{101}$ Series workshop realizes the focus of those events is to leave attendees with a stronger understanding of the regulations and guidelines that all US institutions are expected to follow. As the Founding IACUC Chair at the PHS-assured, AAALAC-accredited animal research program at the Western Michigan University Homer Stryker M.D. School of Medicine (WMed), I recently attended a protocol review session at a national 